\title{
COMPARATIVE RAMAN SPECTROSCOPY STUDY OF THE COELOMIC FLUID OF GRAZING SEA URCHINS AND THEIR NATIVE SEAWATER: PROSPECT FOR A POTENTIAL INDICATOR OF ENVIRONMENTAL AGGRESSION
}

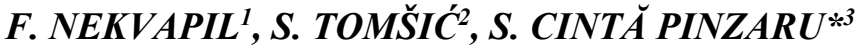

\begin{abstract}
Sea urchins have limited ability to move, and they use the watervascular circulation system to pump surrounding sea water in order to set body parts in motion. It is therefore thought that coelomic fluid, a body fluid of sea urchins which acts as its internal transport and immune system, contains metabolised pollutants. In the present study, we developed a method for detection of carotenoids in the coelomic fluid by Raman spectroscopy. Carotenoids were obtained from the coelomic fluid by ethanol extraction and their selective resonance Raman signal was enhanced employing the surface enhanced Raman scattering (SERS) technique. Carotenoids are ingested by sea urchins through their plant-based diet, metabolised and transported into the coelomic fluid, where they were detected for the first time via SERS. We further investigated the correlations of carotenoids signalling from the coelomic fluid with the local sea water to prospect a potential linkage with changes under environmental aggression. The antioxidative and immunomodulatory role of carotenoids, especially of $\beta$-carotene, was extensively studied in vertebrates (Chew and Park, 2004). Biological defence and increased antioxidant activity is associated with an increased carotenoids level and/or change in their species balance in native sea urchins. Additionally, we compared relative sulfate concentration of sea urchin coelomic fluid with local sea water using FT-Raman technique. We discuss the possibility for development of methods for rapid and cost effective monitoring of the native environmental waters via sea urchin carotenoids. Other co-existent pollutants which may enter the coelomic fluid via the digestive system or through water-vascular system of sea urchins are expected to correlate with the animal response via an increased antioxidant activity due to carotenoids. Thus, sea urchins may prove to be good sentinels of environmental water changes via their carotenoids signalling.
\end{abstract}

Keywords: environmental water, pollutants, sea urchins, Raman spectroscopy.

\section{INTRODUCTION}

Paracentrotus lividus is a common species of sea urchin in the study area, the southeastern Adriatic Sea. The biology and ecology of the species was

\footnotetext{
${ }^{1}$ Babeș-Bolyai University, Faculty of Physics, Str. Mihail Kogălniceanu nr. 1, 400084, Cluj-Napoca, Romania, e-mail: neki.fran@gmail.com

${ }^{2}$ University of Dubrovnik, Department of Aquaculture, Ćira Carića str., nr. 4, 20000 Dubrovnik, Croatia, email: sanja.tomsic@unidu.hr

${ }^{3}$ Corresponding author Babeș-Bolyai University, Faculty of Physics, Str. Mihail Kogălniceanu nr. 1, 400084, Cluj-Napoca, Romania, e-mail: simona.cinta@phys.ubbcluj.ro
} 
extensively reviewed by Boudouresque and Verlaque (2013). Sea urchins are sedentary animals, meaning that their ability to move is limited, and they are exposed to local environmental conditions. According to latter review, the species is not sensitive to organic pollution, it can tolerate and accumulate heavy metals, but it is sensitive to ammonia pollution and oil spills. Since P. lividus is a benthic grazer, it is expected to accumulate pollutants incorporated in algae and marine plants from its surrounding.

The coelomic fluid fills the coelomic cavity of sea urchins. It is a cocktail of various types of cells (Arizza et al., 2007) and biomolecules (Liyana-Pathirana et al., 2002; Dheilly et al., 2013; Dev and Robinson, 2014). Since this fluid serves, inter alia, as the sea urchin`s immune system (reviewed by Ramirez-Gómez and García-Arráras, 2010), we have strong reason to believe that this fluid would reflect chemical and physical changes in the local environment through presence of molecular signs of stress and metabolised xenobiotics.

Carotenoids are a huge class of biomolecules with various biological properties, health benefits and commercial applications. They are usually synthesized by photosynthetic organisms like plants and algae (Vilchez et al., 2011). Sea urchins are not able to synthesize carotenoids de novo, but they possess enzymes which modify the carotenoids ingested through their plant-based diet. They are initially metabolised by the digestive system and then further distributed over the animal's body (Symonds et al., 2007).

In the present paper, we show the first detection of carotenoids in P. lividus coelomic fluid using multiple Raman spectroscopy techniques. Coelomic fluid of this species has not been investigated yet with respect to carotenoids. Carotenoids have previously been identified in sea urchin Strongylocentrotus droebachiensis by means of thin layer chromatography. However, Raman spectroscopy has the advantages of rapidity, simple sample preparation and low per-analysis cost.

\section{MATERIALS AND METHODS}

Five sea urchin individuals of species $P$. lividus were collected from Bay of Gruž, Dubrovnik city, Croatia (42.662884 N, 18.077690 E). A photograph of the collection site is presented in Fig. 1. Collection was done from the shore using a hand held net, and the animals were taken from the depth of about $0.5 \mathrm{~m}$. Additionally, local sea water was sampled in May 2016, and April and June 2017, using plastic 0.51 bottles, from the same site and depth as were the sea urchins.

Coelomic fluid was withdrawn from the coelomic cavity of the animals using syringes. Each sea urchin provided about 2 to $3 \mathrm{ml}$ of coelomic fluid. Coelomic fluid withdrawn from first two sea urchins (about $2 \mathrm{ml}$ each) was discharged into 5-ml sample vials containing $2 \mathrm{ml}$ of phosphate buffer ( $\mathrm{pH} \mathrm{7)}$, and immediately cooled to $4{ }^{\circ} \mathrm{C}$. The fluid from remaining three sea urchins was discharged into respective $10 \mathrm{ml}$ vials for each individual, and the remaining volume till $10 \mathrm{ml}$ was filled with analytical grade ethanol (>99.5\% purity). 
Handling of coelomic fluid from withdrawal to discharge into vials was done fast, in order to avoid premature agglutination inside syringes.

Ethanol extraction lasted 7 days, at ambient temperature and in darkness. Thereafter, respective subsamples were taken from the extraction vials for conventional Raman and for Surface-Enhanced Raman Scattering (SERS) analyses. Raman analyses were done directly in $1 \mathrm{ml}$ glass cuvettes, and SERS solutions of both native fluid (in phosphate buffer) and ethanol extracts were prepared by adding $10 \mu 1$ of each coelomic fluid sample to $500 \mathrm{ml} \mu \mathrm{l}$ of colloidal silver nanoparticles (AgNPs).
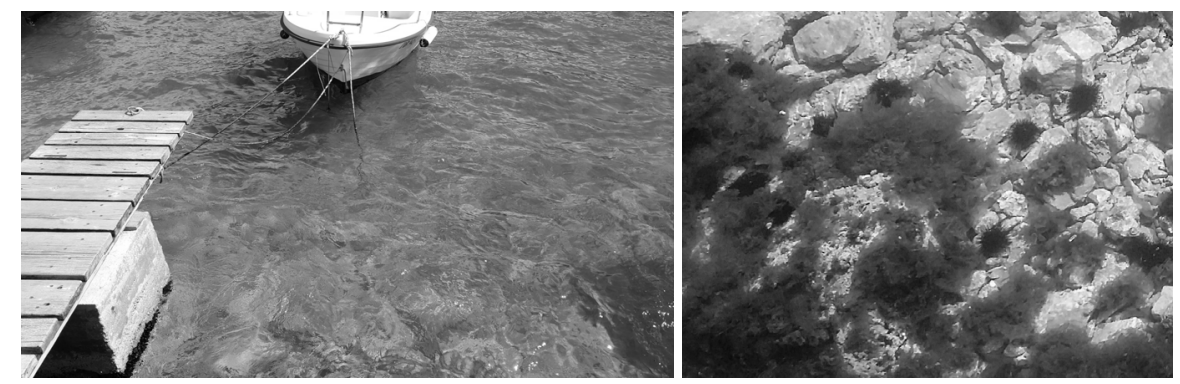

Fig. 1. Photographs of the collection site, showing the shallow rocky sea bottom covered with algae and inhabited by sea urchins.

Raw sea water was filtered on a Milipore vacuum filtration system. The entire volume $(0.5 \mathrm{l})$ was passed through a carbon fibre filter with $0.45 \mu \mathrm{m}$ pore size. Sea water samples were kept at $4{ }^{\circ} \mathrm{C}$ during storage. SERS solutions were prepared by addition of $10 \mu \mathrm{l}$ of filtered sea water into $500 \mu \mathrm{l}$ of AgNPs.

Details on preparation and characterisation of AgNPs can be found in a paper by Cintă Pinzaru et al. (2016). In short, AgNPs were prepared by the LeeMeisel method: silver nitrate reduction by trisodium citrate under boiling temperature. Nanoparticle quality was checked by UV-Vis absorption spectroscopy.

Raman spectroscopy analyses of coelomic fluid extracts were done on a Renishaw InVia confocal Raman microscope, using accessory equipment for liquid samples. Spectra were acquired using a Cobolt diode pumped solid state (DPSS), air cooled laser emitting at $532 \mathrm{~nm}$. The system was operated through WiRE 3.4 software, and the spectra were acquired with $0.5 \mathrm{~cm}^{-1}$ spectral resolution.

Raman spectroscopy analyses of native coelomic fluid and filtered sea water samples were done on a DeltaNu Advantage 532 compact, dispersive Raman spectrometer. A $532 \mathrm{~nm}$ laser line was employed for sample excitation, and spectra were recorded with $8 \mathrm{~cm}^{-1}$ spectral resolution through NuSpec software.

Fourier transform Raman spectroscopy (FT-Raman) spectra were collected with a Bruker Equinox 55 FT-IR spectrometer with an integrated FRA 106S Raman module. A Nd:YAG laser emitting at $1064 \mathrm{~nm}$ with the output power of $350 \mathrm{~mW}$ was used for FT-Raman spectra excitation. Spectra were acquired with 
500 accumulations and spectral resolution of $4 \mathrm{~cm}^{-1}$. Origin 6.1 software was used for processing of spectral data.

\section{RESULTS}

\subsection{Surface-enhanced Raman scattering}

Applying SERS with $10 \mu \mathrm{l}$ of native coelomic fluid added to $500 \mu \mathrm{l}$ AgNPs results in strong signal dominated by the bands at 1563 and $1369 \mathrm{~cm}^{-1}$, along with other medium or weak bands, as showed in the Fig. 2A.

The two strong bands observed in the SERS feature of coelomic fluid are believed to the due to the skeletal structure of echinochrome, the naphthoquinoid pigment from sea urchins. Echinochrome is known to be responsible for the antibactericidal activity in European edible sea urchin (E. esculentus) (Service and Wardlaw, 1984). Although the vibrational properties of echinochrome $(2,3,5,6,8$ pentahydroxy-7-ethyl-1,4-naphthoquinone, PubChem CID: 164644) are not reported in the literature, naphthalene-dione fused rings stretching modes of naphthoquinone and 2-methyl naphthoquinone derivative and their radical anions have been extensively investigated (Singh et al., 2010). The additional five hydroxyl groups in echinochrome are not expected to drastically change the skeletal vibrational structure relative to that of ethyl-1,4-naphtoquinone.

Lorentzian fit of the $1500-1640 \mathrm{~cm}^{-1}$ range from the SERS spectrum of coelomic fluid featuring the main band at $1563 \mathrm{~cm}^{-1}$ showed a complex contribution of seven components (Fig. 2B). Among them, two distinct bands attributable to carotenoids are observed at 1524 and $1532 \mathrm{~cm}^{-1}$. The carotenoid band at $1524 \mathrm{~cm}^{-1}$ could be tentatively assigned to echinenone, while the band at $1532 \mathrm{~cm}^{-1}$ could be originated from fucoxanthin species. Given their low intensity in SERS (combined with the resonance Raman effect), it is assumed that the concentration of carotenoids in coelomic fluid sample is extremely low.

SERS spectra of coelomic fluid extracts in ethanol featured even more defined carotenoid bands (Fig. 2C). The three main carotenoid Raman bands, caused by major atomic vibrations within the molecule, are termed $v_{1}, v_{2}$ and $v_{3}$. The $v_{1}$ band, positioned at $1527 \mathrm{~cm}^{-1}$, is the strongest band, and it is caused by $\mathrm{C}=\mathrm{C}$ double bond stretching from the conjugated polyene chain of carotenoids. Multiple carotenoid species may contribute to the band profile. The $v_{2}$ band at $1157 \mathrm{~cm}^{-1}$ arises from single $\mathrm{C}-\mathrm{C}$ bonds in the polyene chain, while the $v_{3}$ band, which is mainly caused by methyl rocking, was clearly split into two peaks at 1000 and $1013 \mathrm{~cm}^{-1}$ (Tschirner et al., 2009; Cintă Pinzaru et al., 2015).

On the other hand, conventional Raman spectra of native coelomic fluid featured a high fluorescence background, and no carotenoid bands could be observed (Fig. 2A). Similarly, the ethanol extracts yielded normal Raman spectrum of stronger ethanol bands and a weaker carotenoid signal (Fig. 2C).

Comparison of the SERS signal of filtered seawater from the sea urchin environment with that from the coelomic fluid showed a slightly different 
carotenoids contribution (bands at 1523 and $1533 \mathrm{~cm}^{-1}$ ) (Fig. 2D). These results suggests that the environmental water contribution to the SERS output of coelomic fluid is not exclusive, chemical metabolites being present in the latter. The fit components revealing the carotenoids bands are quite different.
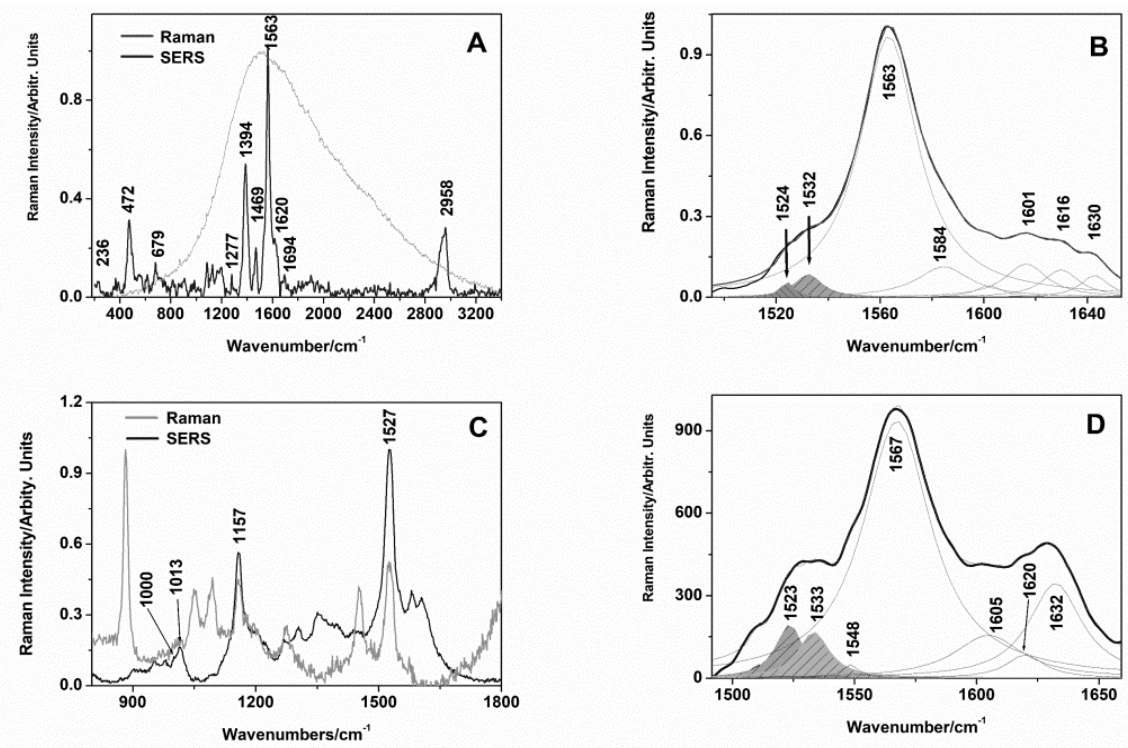

Fig. 2. A) Raman and SERS spectra collected from native P. lividus coelomic fluid; B) Lorentzian fit of the 1500-1650 $\mathrm{cm}^{-1}$ range, with the main echinochrome band at 1563 $\mathrm{cm}^{-1}$ showing carotenoid components at 1524 and $1532 \mathrm{~cm}^{-1}$;C) Raman spectra of ethanolic extract from P. lividus coelomic fluid, showing the ethanol bands along with the carotenoids signal, as indicated; D) Lorentzian fit of the filtered sea water SERS band at $1567 \mathrm{~cm}^{-1}$ showing carotenoid components at 1523 and $1533 \mathrm{~cm}^{-1}$. Excitation: $532 \mathrm{~nm}$.

\subsection{Fourier transform Raman spectroscopy}

FT-Raman spectra of $P$. lividus coelomic fluid compared to sea water from its native environment is presented in Fig. 3.

The water band at $3245 \mathrm{~cm}^{-1}$ in environmental seawater signal has been used to calculate the relative intensity of the sulfate to water Raman ratio, to estimate the concentration of sulfate in coelomic fluid. Data shown in Fig. 3 indicate that sulfate in coelomic fluid is higher than in environmental sea water. The compared ratio $\mathrm{R}$ of different sea water samples is slightly different, however, consistent with small fluctuation over time. 

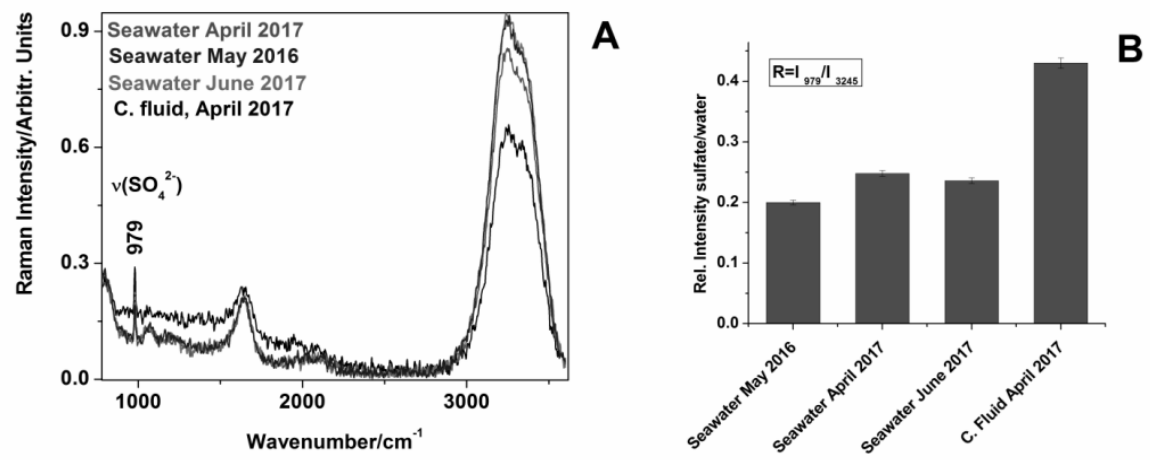

Fig. 3. A: FT-Raman spectra of P. lividus coelomic fluid compared to the raw sea water signal collected from urchin environment. The sulfate Raman band at $979 \mathrm{~cm}^{-1}$ and the water band at $3245 \mathrm{~cm}^{-1}$ were used to calculate the ratio $R$ of sulfate to water plotted in $B$. Excitation: $1064 \mathrm{~nm}$.

\section{DISCUSSION AND CONCLUSIONS}

We have shown that is possible to detect carotenoids and sulfates both in sea urchin coelomic fluid and local environmental water using Raman spectroscopy techniques. It was difficult to record Raman bands of carotenoids using conventional Raman technique and the $532 \mathrm{~nm}$ laser line, because of their low concentration and strong fluorescence of polycyclic aromatic species (echinochrome) which completely covered the carotenoids signal even for the resonance Raman conditions. However, FT-Raman spectroscopy using the NIR laser line at $1064 \mathrm{~nm}$ was able to overcome the fluorescence issue, but the weak carotenoid signal was not detectable in the fluid. Additional information was revealed via the sulfate band from the FT-Raman spectrum: its intensity relative to water was found higher in coelomic fluid than in native seawater. SERS was able to enhance Raman signals from carotenoids present at very low concentration. In addition, the water band at $3425 \mathrm{~cm}^{-1}$ and colloidal AgNPs lattice bands between 100 and $300 \mathrm{~cm}^{-1}$ may serve as internal standards, allowing relative quantification of dissolved molecular species.

The carotenoid profile of $P$. lividus coelomic fluid preserved in phosphate buffer, partially uncovered by Raman spectroscopy in the present study, is similar to the profile of $S$. droebachiensis, where Liyana-Pathirana et al. (2002) reported fucoxanthin as the most abundant carotenoid and minor quantities of xanthophylls (astaxanthin, canthaxanthin).

Based on the corroborated results of these experiments, we suggest that the carotenoid profile of the coelomic fluid and their relative concentration could be used as indicators of environmental water status. Further monitoring studies are certainly needed. First step in developing such indicators would be to calculate their average values and establish the natural range and seasonal variation. Thereafter, statistically relevant number of samples could draw the "normal values", and any significant 
deviation will indicate that the individual is under stress, and may in turn provide grounds for deeper research into the status of the environment.

This study shows that analysis of coelomic fluid is not straightforward: detection of carotenoids required the use of SERS, and detection of sulfate in coelomic fluid required the use of FT-Raman spectroscopy with an infrared laser line. However, in both cases we have achieved our goal, proving that coelomic fluid reveals a Raman signature of seawater and a SERS signature of metabolic carotenoids. These findings suggest that optimized, sensitive detection methods based on Raman spectroscopy could be employed in fast tracking any occurrence environmental chemistry changes in native waters inhabited by sea urchins.

\section{REFERENCES}

1. Arizza, V., Giaramita, F.T., Parrinello, D., Cammarata, M., Parrinello, N. (2007), Cell cooperation in coelomocyte cytotoxic activity of Paracentrotus lividus coelomocytes. Comp. Biochem. Phys. A 147, 389-394.

2. Boudouresque, C.F., Verlaque, M. (2013), Paracentrotus lividus. In: Lawrence, J.M. (Ed.) Sea urchin: Biology and Ecology. Developments in Aquaculture and Fisheries Science 38, 297-328.

3. Chew, B.P., Park, J.S. (2004), Carotenoid action on the immune response. J. Nutr. 134(1), 257S-261S.

4. Cintă Pinzaru, S., Müller, Cs., Tomšić, S., Venter, M.M., Cozar, B.I., Glamuzina, B. (2015), New SERS feature of $\beta$-carotene: consequences for quantitative SERS analysis. J. Raman Spectrosc. 46: 597-604.

5. Cintă Pinzaru, S., Müller, Cs, Tomšić, S., Venter, M.M., Brezeștean, I., Ljubimir, S., Glamuzina, B. (2016), Live diatoms facing Ag nanoparticles: surface enhanced Raman scattering of bulk Cylindrotheca closterium pennate diatoms and of the single cells. RSC Adv. 6, 42899-42910.

6. Dev, S. Robinson, J.J. (2014), Comparative biochemical analysis of the major yolk protein in the sea urchin egg and coelomic fluid. Develop. Growth Differ. 56, 480490.

7. Dheilly, N.M., Raftos, D.A., Haynes, P.A., Smith, L.C., Nair, S.V. (2013), Shotgun proteomics of coelomic fluid from the purple sea urchin, Strongylocentrotus purpuratus. Dev. Comp. Immunol. 40, 35-50.

8. Liyana-Pathirana, C., Shahidi, F., Whittick, A. (2002), Comparison of nutrient composition of gonads and coelomic fluid of green sea urchin Strongylocentrotus droebachiensis. J. Shellfish Res. 21(2): 861-870.

9. Ramírez-Gómez \& F., García-Arrarás, J.E. (2010), Echinoderm immunity. Invertebrate Survival Journal 7, 211-220.

10. Service, M., Wardlaw, A.C. (1984), Echinochrome-A as a bactericidal substance in the coelomic fluid of Echinus esculentus (L.). Comp. Biochem. Phys. 79(2), 161-165.

11. Singh, P., Singh, N.P., Yadav, R. (2010), Vibrational study on the molecular structure of 1,4-naphthoquinone and 2-mathyl-1,4-naphthoquinone and their radical anions by using density functional theory. J. Chem. Pharm. Res. 2(6), 199-224.

12. Symonds, M.C., Kelly, M.S., Caris-Veyrat, C., Young, A.J. (2007), Carotenoids in the sea urchin Paracentrotus lividus: Occurrence of 9 '-cis-echinenone as the dominant carotenoid in gonad colour determination. Comp. Biochem. Phys. B 148, 432-444. 
13. Tschirner, N., Schenderlein, M., Brose, K., Schlodder, E., Mroginski, M.A., Thomsen, C., Hildebrandt, P. (2009), Resonance Raman spectra of $\beta$-carotene in solution and in photosystems revisited: an experimental and theoretical study. Phys. Chem. Chem. Phys. 11, 11471-11478.

14. Vilchez, C., Forján, E., Cuaresma, M., Bédmar, F., Garbayo, I., Vega, J.M. (2011), Marine carotenoids: Biological functions and commercial applications. Mar. Drugs 9, 319-333. 